\title{
Präzises Abformen auch in Extremsituationen
}

\section{Für die Anfertigung von passgenauem Zahnersatz ist eine ex- akte Abformung der präparierten Zähne im Mund, auch unter ungünstigen Voraussetzungen, erforderlich. Die modernen Produkte der Firma Kettenbach in Form der A-Silikone Panasil ${ }^{\circledR}$ binetics Putty Fast als Trägermaterial und Panasil ${ }^{\circledR}$ initial con- tact X-Light als hydrophiles Korrekturmaterial sind dazu her- vorragend geeignet.}

\section{Die Korrekturabformung im Praxistest}

Bei der Abformung präparierter Stümpfe im Mund des Patienten stellt sich auch für jeden erfahrenen Prothetiker ein immer wiederkehrendes Problem insofern, als es trotz aller Anstrengung bei der Trockenlegung mit den Retraktionsfäden, Watterollen und der Absaugung zur Speichelbenetzung der Stümpfe kommt. Mit diesem Problem wird das Korrekturmaterial Panasil initial contact X-Light mit seiner nachweislich hydrophilen Struktur sehr gut fertig. Diese Struktur bleibt darüber hinaus während der gesamten Verarbeitungszeit erhalten. Deshalb haben uns die Abdruckmaterialien im Praxistest eindrucksvoll überzeugt.

Für die Korrekturabformung haben wir in der Praxis als Basisträgermaterial Panasil binetics Putty Fast aus der komfortablen Großkartusche ausgebracht. Die Dosierung ist genau und der Materialverbrauch ist gering. Das Korrekturmaterial Panasil initial contact X-Light wird mit der Applyfix-Dosierpistole angewendet und ist mit allen Trägermaterialien der Panasil-Produktreihe kombinierbar.

In unserem Fall wurde eine Brücke im 2. Quadranten vorbereitet. Nach der Pfeilerpräparation legen wir Retraktionsfäden, um den Sulkus aufzuhalten und die Blutung zu stillen ( $\bullet$ Abb.1). Die Stümpfe werden danach trocken geblasen und sind nun vorbereitet für die Vorabformung.

Zur Abformung verwenden wir einen Rimlocklöffel. Die Befüllung des Löffels

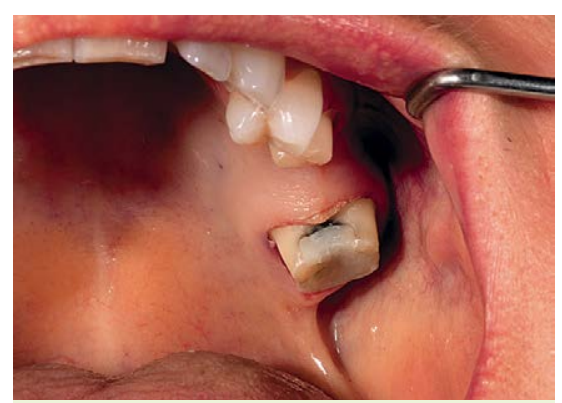

Abb. 1. Nach Entfernung der Retraktionsfäden sind die Präparationsgrenzen dargestellt.

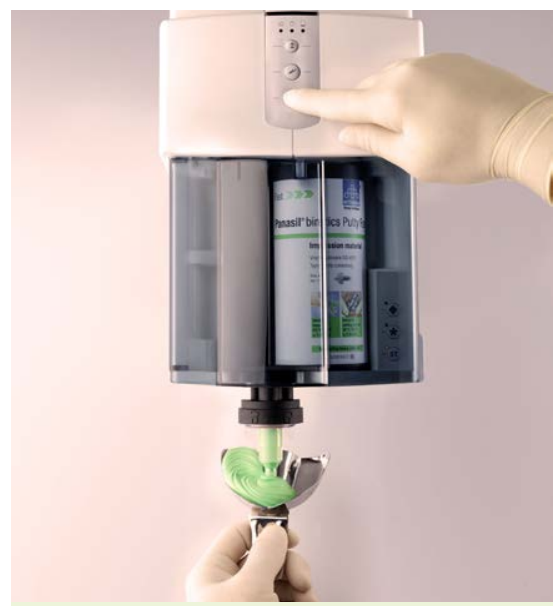

Abb. 2 Befüllung des Rimlocklöffels mit Panasil binetics Putty Fast von Kettenbach.

erfolgt aus der Großkartusche von Kettenbach ( Abb. 2). Das absolut homogen gemischte Material wird auf den Löffel von einer Seite zur anderen aufgefüllt und kurz glattgedrückt. Mit dem Panasil binetics Putty Fast haben wir 1,5 min Zeit, um den Löffel mit sanftem Druck im Mund des Patienten zu platzieren - für unser eingespieltes Team kein Problem.
Die Korrekturabformung -

Hydrophilie führt uns zum Ziel $\nabla$

Nach 2,5 min wird der Löffel entfernt. Im Rand- und Gaumenbereich wird der Abdruck zurückgeschnitten und dann im Mund des Patienten zur Passprobe reponiert und anschließend trocken geblasen (o Abb. 3).

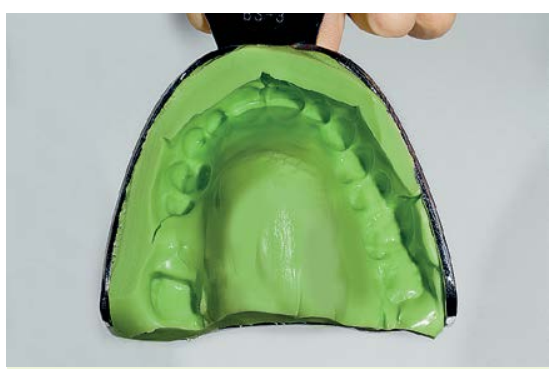

Abb. 3 Zurückgeschnittener Vorabdruck.

Für die Korrekturphase kommt das hydrophile Panasil initial contact X-Light im Kartuschensystem zum Einsatz. Mit einem dünnen Aufsatz auf dem statischen Mischer wird der Sulkus der Zähne nach Entfernung der Fäden aufgefüllt. Anschließend wird der vorbereitete Löffel mit dem gleichen Material bestückt. Lufteinschlüsse werden vermieden durch Halten der Spitze im Abdruckmaterial ( 0 Abb. 4+5).

\section{Das Ergebnis in unserem Patien- tenfall $\nabla$}

Das dünnfließende Korrekturmaterial hat trotz Restfeuchte alle Sulkusbereiche exakt ausgefüllt. Die Benetzung der Zahnoberfläche und das gute Einfließen in den Sulkus ist auf die hohe initiale Hydrophilie zurückzuführen und von der 1. Sekunde an gewährleistet. Nach dem Einbringen ist es sehr standfest. In unserem Patientenfall handelt es sich um eine Abformung im Oberkiefer und obwohl die Konsistenz des Korrekturmaterials sehr dünnfließend ist, verharrt es an Ort und Stelle und tropft nicht ab. Alle Panasil-Materialien haben ein temperaturgesteuertes Abbindeverhalten. Auch bei umfänglichen prothetischen Arbeiten ist eine stressfreie Applikation in der Gesamtverarbeitungszeit möglich. Temperaturen, die erheblich über $23^{\circ} \mathrm{C}$ liegen, verkürzen natürlich die Gesamtverarbeitungszeit im Mund. Vorabdruck- und Korrekturmaterial werden von den Patienten geschmacksmäßig als neutral bezeichnet und die Löffel lassen sich gut aus dem Mund entfernen. 


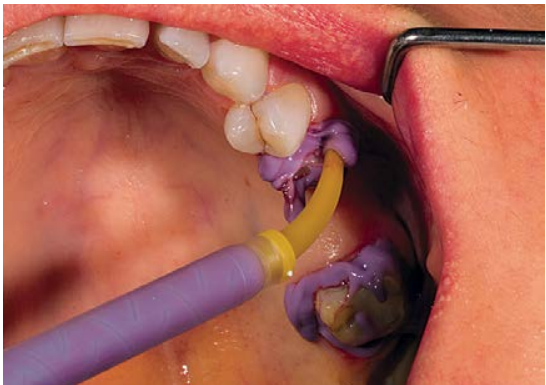

Abb. 4 Umspritzens der präparierten Stümpfe mit dem dünnfließenden, lilafarbenen Panasil initial contact X-Light. Auch bei Restfeuchte zeigt sich ein gutes Fließverhalten und Standfestigkeit im Oberkiefer. Kein Abtropfen! Die Zahnoberflächen werden sofort und bleibend benetzt.

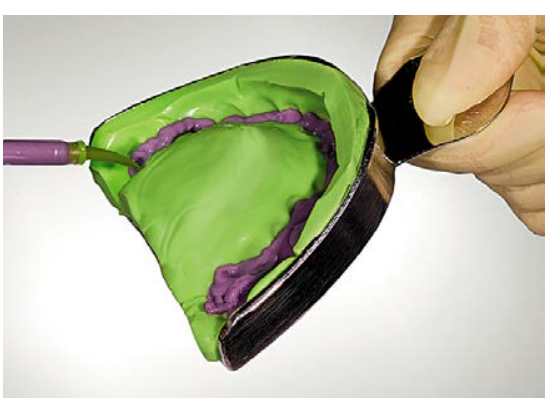

Abb. 5 Befüllung der Vorabformung mit dem dünnfließenden Panasil initial contact X-Light vor dem Einbringen.

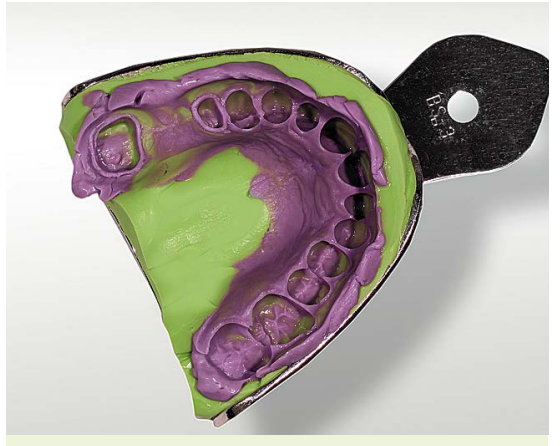

Abb. 6 Gute Detailzeichnung der Präparationsgrenzen und Septen sowie stabiler Verbund von Korrekturmaterial und Trägersubstanz.

\section{Exakte und zeichnungsscharfe $\mathbf{A b}$ - formung}

Die Beurteilung der Abformung lässt sich aufgrund des Farbkontrasts zum Trägermaterial gut nachvollziehen. Durch die Reißfestigkeit werden auch feine Strukturen und Interdentalsepten gut abgebildet. Der Haftverbund zwischen beiden Abdruckmaterialien ist hervorragend und die Zeichnungsschärfe ist aufgrund der Hydrophilie als sehr gut anzusehen. Die Hydrophilie hat auch im zahntechnischen Labor Vorteile. Die Abdrücke müssen nicht mit Entspannungsmitteln bearbei- tet werden und können wegen der hohen Reißfestigkeit mehrmals erfolgreich ausgegossen werden (० Abb.6).

\section{Hohe Präzision und angenehme Verarbeitung \\ $\nabla$}

Kettenbach hat mit dem Panasil binetics Putty Fast und dem dazugehörigen Korrekturmaterial ein hervorragend aufeinander abgestimmtes System zum Darstellen von allen nur denkbaren Situationen im Patientenmund geliefert. Dank der extremen Hydrophilie und guten Benetzung sowie der Standfestigkeit des Korrekturmaterials lassen sich alle Situationen in den Griff bekommen. Hervorzuheben sind auch die beispielhafte Einbringung der Materialien mit dem Plug \& Press ${ }^{\circledR}$-System und die sichere Applikation des Korrekturmaterials mittels Kartuschensystem und passenden Applikationen.

\section{Kontakt}

Dr. Manfred Simaitis

Grevenbroich

Praxis-Simaitis-Ventocilla@t-online.de

Dieser Beitrag entstand mit freundlicher Unterstützung der Kettenbach GmbH \& Co. KG, Eschenburg 